\title{
The impact of age on propofol requirement for inducing loss of consciousness in elderly surgical patients
}

\author{
Hua Yang ${ }^{1}$, Hui-Min Deng ${ }^{1}$, Jun-Wei $\mathrm{Ji}^{1}$, Xin Lv${ }^{1}$, and Jin-Chao Song ${ }^{1}$ \\ ${ }^{1}$ Affiliation not available
}

January 19, 2021

\begin{abstract}
Aims: It is generally accepted that geriatric patients are more sensitive to propofol than adults; thus, a dose-adjusted propofol is recommended for these patients during the induction of anesthesia. However, for patients aged 75 years and over, established guidelines do not provide dose references for the anesthesiologists. To this end, we observed 80 surgical patients (female 39 , male 41, American Society of Anesthesiologists physical status score I II) to access the appropriate dose of propofol for inducing loss of consciousness (LOC). Methods: Patients were subdivided into group A (20 patients, 45 64 yr), group B (20 patients, $\left.65^{\sim} 74 \mathrm{yr}\right)$, group C (20 patients, $\left.75^{\sim} 84 \mathrm{yr}\right)$, and group D (20 patients, [?] $\left.85 \mathrm{yr}\right)$. All patients received propofol (at a rate of $0.3 \mathrm{mg} / \mathrm{kg} / \mathrm{min}$ ) alone for inducing LOC, which was defined by loss of both eyelash reflex and verbal response. Results: Compared with group A, the propofol requirement for LOC in Group B, C and D decreased by $14.8 \%, 25.2 \%$ and $38.5 \%$, respectively. Bivariate linear correlation analysis showed that propofol requirement was negatively correlated with age. After adjusting for potential confounders, age was still an independent factor affecting propofol requirement. Conclusion: The propofol requirement for inducing LOC decreased significantly in elderly patients. We demonstrated that age was an independent factor impacting propofol requirement for LOC during the induction of general anesthesia, implying that the propofol dose for anesthesia induction should be further reduced in elderly surgical patients, especially those aged 75 years and over.
\end{abstract}

The impact of age on propofol requirement for inducing loss of consciousness in elderly surgical patients

Running Title : Impact of age on propofol requirement

Hua Yang ${ }^{1}$, Hui-Min Deng ${ }^{2}$, Jun-Wei $\mathrm{Ji}^{1}, \mathrm{Xin} \mathrm{Lv}^{2}$, Jin-Chao Song ${ }^{1}$

${ }^{1}$ Department of Anesthesiology, Shidong Hospital of Shanghai, University of Shanghai for Science and Technology, Shanghai, China.

${ }^{2}$ Department of Anesthesiology, Shanghai Pulmonary Hospital, Tongji University

School of Medicine, Shanghai, China.

Hua Yang, Hui-Min Deng and Jun-Wei Ji contributed equally to this work.

Corresponding Author: Jin-Chao Song, M.D., Department of Anesthesiology, Shidong Hospital of Shanghai, University of Shanghai for Science and Technology, 999 Shiguang Road, Shanghai, China. Phone: (86) 21-25066782. Email: sjch2013@163.com; Xin Lv, M.D. Department of Anesthesiology, Shanghai Pulmonary Hospital, Tongji University School of Medicine, Shanghai, 200433, China. E-mail: jokul2020@sohu.com.

The authors confirm that the Principal Investigator for this paper isJin-Chao Song and that he had direct clinical responsibility for the patients.

Key words: elderly, anesthetics, propofol, intravenous anaesthesia, loss of consciousness 
Word count : abstract (243), main text (2500)

Table count : 4

Figure count : 2

\section{Abstract}

Aims: It is generally accepted that geriatric patients are more sensitive to propofol than adults; thus, a dose-adjusted propofol is recommended for these patients during the induction of anesthesia. However, for patients aged 75 years and over, established guidelines do not provide dose references for the anesthesiologists. To this end, we observed 80 surgical patients (female 39, male 41, American Society of Anesthesiologists physical status score I II) to access the appropriate dose of propofol for inducing loss of consciousness (LOC).

Methods: Patients were subdivided into group A (20 patients, 45 64 yr), group B (20 patients, 65 74 yr), group C (20 patients, 75 $84 \mathrm{yr}$ ), and group D (20 patients, [?] $85 \mathrm{yr}$ ). All patients received propofol (at a rate of $0.3 \mathrm{mg} / \mathrm{kg} / \mathrm{min}$ ) alone for inducing LOC, which was defined by loss of both eyelash reflex and verbal response.

Results: Compared with group A, the propofol requirement for LOC in Group B, C and D decreased by $14.8 \%, 25.2 \%$ and 38.5\%, respectively. Bivariate linear correlation analysis showed that propofol requirement was negatively correlated with age. After adjusting for potential confounders, age was still an independent factor affecting propofol requirement.

Conclusion: The propofol requirement for inducing LOC decreased significantly in elderly patients. We demonstrated that age was an independent factor impacting propofol requirement for LOC during the induction of general anesthesia, implying that the propofol dose for anesthesia induction should be further reduced in elderly surgical patients, especially those aged 75 years and over.

\section{What is already known about this subject:}

1. Geriatric patients are more sensitive to propofol than adults.

2. Established guidelines do not provide dose references for patients aged 75 years and over.

3. We found that elderly patients commonly receive greater-than-recommended doses of propofol for the induction of general anesthesia.

\section{What this study adds:}

1. The impact of age on propofol requirement for inducing loss of consciousness in elderly surgical patients are demonstrated.

2. The propofol dose for anesthesia induction should be further reduced in elderly surgical patients, especially those aged 75 years and over.

\section{Introduction}

Advances in surgical techniques and improvement of perioperative management have led to a larger proportion of elderly patients presenting to undergo surgical procedures [1]. Previous studies have shown that more than half of all surgical procedures were performed on patients over the age of 65 [2]. With the deepening of global aging, this proportion is expected to further increase in the coming decades. For these patients, anesthesiologists often need to adjust the anesthetic regimen, including medication selection, dosage optimization and so on, to adapt to the elderly physiological changes. However, established guidelines on anesthesia do not provide dose references for the anesthesiologists' clinical practice [3].

Propofol, as an intravenous hypnotic agent, has been widely used for anesthetic induction and maintenance in surgical patients. It can provide quick and smooth anesthesia induction. However, a common sideeffect of propofol-based induction is dose-dependent hemodynamic instability [4], such as hypotension and 
bradycardia, especially in elderly patients. Compared with the middle-aged adult, the principles of geriatric physiology are not merely a linear extension [1]. These elderly patients represent a unique clinical group, in addition, they typically suffer from a number of chronic diseases. Although some studies have recommended that propofol-based induction should be avoided in these elderly individuals [3, 5], many anesthesiologists still choose to use propofol in clinical anesthesia rather than other drugs which have little effect on hemodynamics.

Based on the fact that elderly patients have increased sensitivity to propofol, anesthesiologists are recommended to reduce the dose of propofol used for induction in patients aged over 65 years from $2-2.5 \mathrm{mg} / \mathrm{kg}$ to $1-1.5 \mathrm{mg} / \mathrm{kg}$ [6]. However, for patients over 75 or even 85 years old, it is unclear whether this recommendation is still applicable and what dose of propofol is appropriate for such patients. To this end, we designed the current study to access the appropriate dose of propofol for these patients, and to analyze the role of age on propofol requirement in the process of loss of consciousness (LOC) induced by propofol.

\section{Patients and Methods}

\section{Participants}

We conducted a prospective observational study following the Declaration of Helsinki from April to August 2020. The study was approved by the Institutional Research Ethics Committee of Shidong Hospital. Patients aged 45 years and over scheduled for general surgery or orthopedic surgery under general anesthesia were considered eligible. Patients were excluded if they: American Society of Anesthesiologists (ASA) physical status score [?]3; allergic to propofol; body mass index (BMI) [?] 20 or [?] $30 \mathrm{~kg} / \mathrm{m}^{2}$; taking hypnotics, opioid analgesic or antianxiety agents; known or suspected heart failure (ejection fraction $<40 \%$ ), severe respiratory disease, renal or metabolic diseases; could not complete the informed consent procedure independently. Written informed consent was obtained from all patients.

\section{Study protocol}

A total of 80 patients who met the inclusion and the exclusion criteria were divided into 4 groups, Group A (20 patients, 45 $64 \mathrm{yr}$ ), Group B (20 patients, 65 74 yr), Group C (20 patients, $75^{\sim} 84 \mathrm{yr}$ ), and Group D (20 patients, [?] $85 \mathrm{yr}$ ), according to age. Noninvasive blood pressure, heart rate, electrocardiogram, pulse oxygen saturation and end-tidal carbon dioxide were monitored continuously throughout the operation. After 5 minutes of preoxygenation, propofol was pumped at a rate of $0.3 \mathrm{mg} / \mathrm{kg} / \mathrm{min}$ until the LOC occurred. The LOC was defined by loss of both eyelash reflex and verbal response. The assessment of the loss of eyelash reflex and verbal response was carried out every 10 seconds after propofol pumping for $1.5 \mathrm{~min}$. An anesthesiologist assistant, who was blinded to the grouping, performed the above reflex assessment and finally determined the end point of titration. Meanwhile, the dose of propofol (Propofol requirement) and the time of reflection disappear (T reflection disappear) for each patient were recorded. After induction of propofol, $0.4^{\sim} 0.6 \mathrm{ug} / \mathrm{kg}$ of sufentanil and $0.2 \mathrm{mg} / \mathrm{kg}$ of cisatracurium were administered, and endotracheal intubation was performed three minutes later.

Perioperative variables included in the analysis were sex, BMI, albumin, bilirubin, alanine aminotransferase (ALT), aspartate aminotransferase (AST), serum creatinine (Scr), blood urea nitrogen (BUN), glomerular filtration rate (GFR) and ejection fraction (EF).

Hemodynamic parameters including mean arterial pressure (MAP) and heart rate (HR) at 5 different time points (T0, before propofol administration; T1, LOC; T2, 3 minutes after the administration of fentanyl and cisatracurium; T3, 1 minute after intubation; T4, 5 minutes after intubation; T5, 10 minutes after intubation) were recorded.

Statistical analysis

Baseline characteristics of patients were described as mean (standard deviation, SD) for continuous variables, frequency (percentage) for categorical variables, or median (interquartile range, IQR) for continuous variables with skewed distribution. One-way analysis of variance, Kruskal-Wallis test and $\chi^{2}$ test were used to analyze the demographic data and hemodynamic changes in each group, as appropriate. For trend analyses, we used 
the Mantel-Haenszel $\chi^{2}$ test of linear association for categoical variables and linear regression for continuous variables. Pearson correlation coefficients were calculated to assess correlations between propofol requirement and different parameters.

To evaluate the independent association of age with propofol requirement, multiple linear regression models were constructed. Three models were fitted, Model I: unadjusted; Model II: adjusted for gender and BMI; Model III: further adjusted for albumin, bilirubin, ALT, AST and GFR. Collinearity diagnostics was used to determine whether the variables in models were highly intercorrelated. In addition, we also use smooth curve fitting to examine whether the relationship between age and propofol requirement was linear while adjusting for potential confounders above. All statistical analyses were performed using SPSS, version 20.0 (SPSS Inc., Chicago, IL, USA) and R, version 3.6.3 (R Project for Statistical Computing). A two-side $P$ value $<0.05$ was considered statistically significant.

\section{Results}

The baseline characteristics of patients are presented in Table $\mathbf{1}$. Of the 80 patients (mean age $=74 \pm 12$ years, range $45^{\sim} 93$ years), 39 (48.75\%) were female. No differences in BMI, ALT, AST, BUN, and EF between age groups. However, GFR was significantly associated with age $(\mathrm{P}=0.014)$ and the mean difference $(95 \% \mathrm{CI})$ in age between groups A and B was 29.59 (4.60 54.58). Furthermore, GRF and albumin decreased linearly with age, whereas Scr and bilirubin were reversed (all P-trends $<0.05$ ).

Differences in anesthesia effects between ages were shown inTable 2 . Propofol requirement and $\mathrm{T}$ reflection disappear differed significantly between ages $(\mathrm{P}<0.001)$, and all had significant linear decreases with age (P-trend $<0.001)$. Compared to group A, group B had a mean reduction in propofol requirement of 0.20 $\mathrm{mg} / \mathrm{kg}\left(95 \% \mathrm{CI}=0.07^{\sim} 0.33\right)$ and a mean reduction in $\mathrm{T}$ reflection disappear of $32.50 \mathrm{~s}(95 \% \mathrm{CI}=7.28$ 57.72). Significant differences were consistent across all neighboring groups (all $\mathrm{P}<0.05$ ). Additionally, to further determine whether the relation between age and propofol requirement was linear, the estimated dose-response curve was fitted. There was a continuous linear decreasing trend and statistical significance between propofol requirement and age after adjusting for gender, BMI, albumin, bilirubin, ALT, AST, and GFR (Figure 1 ).

Bivariate linear correlation analysis showed that propofol requirement was significantly and positively correlated with albumin $(\mathrm{r}=0.312 ; 95 \% \mathrm{CI}=0.099 \sim 0.497 ; \mathrm{P}=0.005)$ and $\mathrm{GRF}(\mathrm{r}=0.286 ; 95 \% \mathrm{CI}=0.070$ $0.476 ; \mathrm{P}=0.010$ ), and negatively correlated with age, such that significant decline in propofol requirement with increasing age $\left(\mathrm{r}=-0.689 ; 95 \% \mathrm{CI}=-0.789^{\sim}-0.553 ; \mathrm{P}<0.001\right)$. Other kidney and liver parameters were not significantly correlated with propofol requirements (Table 3 ).

Patient age was an independent and significant factor in propofol requirement. When propofol requirement entered models as a continuous variable, advanced age was associated with lower propofol requirements. In the fully adjusted model, each 1-SD increase in age was associated with a decrease in propofol requirement of approximately $0.171(\beta=-0.170 ; 95 \% \mathrm{CI}=-0.224 \sim-0.117 ; \mathrm{P}<0.001)$. In the unadjusted model, a high level of age (Group D) was strongly associated with a lower propofol requirement $(\beta=-0.525 ; 95 \% \mathrm{CI}=$ $\left.-0.648^{\sim}-0.403 ; \mathrm{P}<0.001\right)$. Furthermore, $\mathrm{P}$-trend was calculated using age groups as ordinal variables, and the results showed a linear trend between age and propofol requirement $(\mathrm{P}$-trend $<0.001)$. The association yielded relatively consistent results after adjusting for gender and BMI [ $\beta$ (95\%CI): Group B: -0.185 (-0.310 $\sim$-0.060), P = 0.004; Group C: -0.348 (-0.470 -0.226), P < 0.001; Group D: -0.512 (-0.637 -0.387$), \mathrm{P}<$ 0.001] (Model II). After further adjustment for albumin, bilirubin, ALT, AST, and GFR, the associations were slightly weakened but still statistically significant, with $\beta$ values of $-0.158\left(95 \% \mathrm{CI}=-0.289^{\sim}-0.027\right.$; $\mathrm{P}$ $=0.019),-0.321\left(95 \% \mathrm{CI}=-0.457^{\sim}-0.184 ; \mathrm{P}<0.001\right)$ and $-0.467\left(95 \% \mathrm{CI}=-0.608^{\sim}-0.327 ; \mathrm{P}<0.001\right)$ for groups B, C, and D, respectively (Model III) (Table 4 ).

After induction of anesthesia, the MAP and HR of patients in each group began to decrease, especially at T2 (3 minutes after the administration of fentanyl and cisatracurium). After intubation, MAP and HR rebounded in different degrees and tended to be stable in $5^{\sim} 10$ minutes (Figure 2 ). However, there was no difference in percent changes relative to the baseline between the 4 groups (MAP: T1, $\mathrm{P}=0.404$; T2, 
$\mathrm{P}=0.558 ; \mathrm{T} 3, \mathrm{P}=0.460 ; \mathrm{T} 4, \mathrm{P}=0.202 ; \mathrm{T} 5, \mathrm{P}=0.109 ; \mathrm{HR}: \mathrm{T} 1, \mathrm{P}=0.499 ; \mathrm{T} 2, \mathrm{P}=0.970 ; \mathrm{T} 3, \mathrm{P}=0.237 ; \mathrm{T} 4$, $\mathrm{P}=0.135 ; \mathrm{T} 5, \mathrm{P}=0.922)$.

\section{Discussion}

In the present study, we investigated the effective dose of propofol in surgical patients aged 45 years and over for LOC during the induction of general anesthesia. We found that the propofol requirement for LOC decreased significantly with increasing age. Additionally, we demonstrated that patient age was an independent and significant factor in propofol requirement for LOC, implying that the propofol dose for anesthesia induction should be further reduced in elderly surgical patients, especially those aged 75 years and over.

Increasing aging population paired with age-associated coexisting diseases and longer life spans have resulted in an increasing proportion of geriatric surgery. For these elderly patients, age-related changes in physiology, anatomy and cognitive function have a great impact on both the pharmacodynamics and pharmacokinetics of administered anesthetics $[1,7,8]$. Anesthesiologists have to tailor the anesthetic scheme to account the changes associated with aging, comorbidities, and patient medications so as to optimize the perioperative prognosis of these elderly patients. However, guiding evidence focusing on geriatric patients remains poor so far. Clinicians tend to adjust the anesthetic regimen according to their own experience. Additionally, a large retrospective cohort study has found that the median (IQR) propofol dose for anesthesia induction in patients aged over 65 years was $1.8\left(1.4^{\sim} 2.2\right) \mathrm{mg} / \mathrm{kg}$, greater than recommended doses $\left(1^{\sim} 1.5 \mathrm{mg} / \mathrm{kg}\right)$ [3]. In our study, the effective dose of propofol for LOC in patients $\left(65^{\sim} 74\right.$ years $)$ was $1.15 \mathrm{mk} / \mathrm{kg}$, which is $14.8 \%$ lower than that for patients aged $45^{\sim} 64$ years. Our findings are in line with previous studies [9, 10]. Moreover, our results show that propofol requirement for LOC in patients aged $75^{\sim} 84$ years and [?] 85 years are $25.2 \%$ and $38.5 \%$ lower than that for patients aged; 65 years, respectively (Table 2 ). Based on age-grouping, we demonstrate that as age increased by decade, the propofol requirement for LOC in elderly reduces dramatically (Figure 1 ). These findings confirm the guideline for minimal administration of propofol and recommend that the dose of propofol for anesthesia induction should be further reduced for patients aged 75 years and over.

One major purpose of this study was to investigate the impact of age on the propofol requirement for LOC in elderly during anesthesia induction. Therefore, we incorporated factors that might have an impact, such as gender, BMI, albumin, bilirubin, ALT, AST, and GFR into the models (Table 4 ). After adjustment for these factors, age was still an independent factor. From the perspective of the increased sensitivity of the elderly to anesthetics, our results do not conflict with previous studies [10-13]. However, unlike previous research paradigms, we focused on elderly surgical population which aged over 65 years, and tried to eliminate the interference caused by concomitant medication, comorbidities and renal insufficiency. Remarkably, this geriatric surgical population represents a special group. They typically suffer from cardiopulmonary dysfunction, metabolic diseases, and nervous system dysfunction, etc. [1, 7, 14]. Increasing age represents the change within senescent process, rather than a sort of pathological condition. Correspondingly, this aging process impedes the ability of the body to maintain homeostasis, especially when the body is under stress $[1,15]$. Meanwhile, studies also have shown that advanced age is an independent risk factor for prognosis of various surgical procedures [16-19]. Therefore, clinicians should be more aware of these changes caused by aging, so as to provide the most effective perioperative treatment for these elderly surgical patients.

There are several limitations in our study. First, this was a single-center observational study, with potential selection biases including race, type of surgery, propofol infusion rate. Second, our study only included patients with $\mathrm{ASAI}^{\sim} \mathrm{II}$, which could eliminate the interference caused by some comorbidities, such as diabetes or hypertension, but it may also limit the universality of our results. Third, each anesthesiologist has his own induction habit such as pretreating with midazolam or dexmedetomidine, which leads to great differences in anesthesia induction.

In addition, some confounding factors that may influence the results might have been overlooked due to unavailable data, including inflammation and nutritional status. 
In conclusion, our pilot observation study in surgical patients aged 45 years and over demonstrated that age was an independent and significant factor in propofol requirement for LOC during the induction of general anesthesia. Propofol dosage should be tailored in elderly patients, especially those older than 75 years, which may eventually bring benefit to these individuals. However, due to the limitations of our research design, further studies are needed to validate this conclusion and to verify whether this will improve the perioperative prognosis of patients.

\section{Acknowledgements}

The authors thank Dr. Ai-Jun You (Tongji University, Shanghai, China) for his help for data analysis.

Authors' contributions : Study design: JCS, XL. Data collection: HY, HMD and JWJ. Statistical analysis: HMD. Manuscript drafting: HMD and JCS. Supervision: XL and JCS. Project administration: JCS.

\section{Funding}

This work was supported by the 2019 Development Fund of Anesthesiology, Shanghai Pulmonary Hospital.

\section{Conflicts of interest}

All authors declare no conflicts of interest.

\section{Data Availability Statement}

The data that support the findings of this study are available from the corresponding author upon reasonable request.

\section{References}

1. Yang R, Wolfson M, Lewis MC. Unique Aspects of the Elderly Surgical Population: An Anesthesiologist's Perspective. Geriatr Orthop Surg Rehabil 2011; 2: 56-64.

2. Veering BT. Management of anaesthesia in elderly patients. Curr Opin Anaesthesiol 1999; 12: 333-6.

3. Phillips AT, Deiner S, Mo Lin H, Andreopoulos E, Silverstein J, Levin MA. Propofol Use in the Elderly Population: Prevalence of Overdose and Association With 30-Day Mortality. Clin Ther 2015; 37: 2676-85.

4. Sahinovic MM, Struys M, Absalom AR. Clinical Pharmacokinetics and Pharmacodynamics of Propofol. Clin Pharmacokinet 2018; 57: 1539-58.

5. Reich DL, Hossain S, Krol M, Baez B, Patel P, Bernstein A, Bodian CA. Predictors of hypotension after induction of general anesthesia. Anesth Analg 2005; 101: 622-8, table of contents.

6. McEvoy MD, Reves JG. Intravenous Hypnotic Anesthetics. In: Geriatric Anesthesiology, edsSilverstein JH, Rooke GA, Reves JG, McLeskey CH, New York, NY: Springer New York, 2008: 229-45.

7. Lim BG, Lee IO. Anesthetic management of geriatric patients. Korean J Anesthesiol 2020; 73: 8-29.

8. Barkin RL, Schwer WA, Barkin SJ. Recognition and management of depression in primary care: a focus on the elderly. A pharmacotherapeutic overview of the selection process among the traditional and new antidepressants. Am J Ther 2000; 7: 205-26.

9. Koh JC, Park J, Kim NY, You AH, Ko SH, Han DW. Effects of remifentanil with or without midazolam pretreatment on the $95 \%$ effective dose of propofol for loss of consciousness during induction: A randomized, clinical trial. Medicine (Baltimore) 2017; 96: e9164.

10. You AH, Kim JY, Kim DH, Suh J, Han DW. Effect of remifentanil and midazolam on ED95 of propofol for loss of consciousness in elderly patients: A randomized, clinical trial. Medicine (Baltimore) 2019; 98: e15132. 
11. Jacobs JR, Reves JG, Marty J, White WD, Bai SA, Smith LR. Aging increases pharmacodynamic sensitivity to the hypnotic effects of midazolam. Anesth Analg 1995; 80: 143-8.

12. Schnider TW, Minto CF, Shafer SL, Gambus PL, Andresen C, Goodale DB, Youngs EJ. The influence of age on propofol pharmacodynamics. Anesthesiology 1999; 90: 1502-16.

13. Minto CF, Schnider TW, Egan TD, Youngs E, Lemmens HJ, Gambus PL, Billard V, Hoke JF, Moore KH, Hermann DJ, Muir KT, Mandema JW, Shafer SL. Influence of age and gender on the pharmacokinetics and pharmacodynamics of remifentanil. I. Model development. Anesthesiology 1997; 86: 10-23.

14. Pickering AN, Hamm ME, Dawdani A, Hanlon JT, Thorpe CT, Gellad WF, Radomski TR. Older Patient and Caregiver Perspectives on Medication Value and Deprescribing: A Qualitative Study. J Am Geriatr Soc 2020; 68: 746-53.

15. Tasch MD. The autonomic nervous system and geriatric anesthesia. Int Anesthesiol Clin 1988; 26: $143-51$.

16. Kazmers A, Perkins AJ, Jacobs LA. Outcomes after abdominal aortic aneurysm repair in those $>$ or $=80$ years of age: recent Veterans Affairs experience. Ann Vasc Surg 1998; 12: 106-12.

17. Keenan CR, White RH. Age as a risk factor for venous thromboembolism after major surgery. Curr Opin Pulm Med 2005; 11: 398-402.

18. Cammarata MJ, Kantar RS, Rifkin WJ, Greenfield JA, Levine JP, Ceradini DJ. Advanced Age Is a Risk Factor for Complications Following Abdominal Panniculectomy. Obes Surg 2019; 29: 426-33.

19. Pommergaard HC, Burcharth J, Rosenberg J, Raskov H. Advanced age is a risk factor for proximal adenoma recurrence following colonoscopy and polypectomy. Br J Surg 2016; 103: e100-5.

\begin{tabular}{|c|c|c|c|c|c|c|}
\hline & $\begin{array}{l}\text { Group A } \\
(\mathrm{n}=20)\end{array}$ & $\begin{array}{l}\text { Group B } \\
(\mathrm{n}=20)\end{array}$ & $\begin{array}{l}\text { Group C } \\
(\mathrm{n}=20)\end{array}$ & $\begin{array}{l}\text { Group D } \\
(\mathrm{n}=20)\end{array}$ & $P$ value & $P$ trend \\
\hline $\begin{array}{l}\text { Male, No. } \\
(\%)\end{array}$ & $9(45)$ & $10(50)$ & $11(55)$ & $11(55)$ & 0.908 & 0.487 \\
\hline $\begin{array}{l}\text { BMI, mean } \\
(\mathrm{SD}), \mathrm{kg} / \mathrm{m}^{2} \\
\text { Liver } \\
\text { function }\end{array}$ & $24.05(0.61)$ & $24.40(0.50)$ & $24.05(0.61)$ & $24.40(0.50)$ & 0.055 & 0.224 \\
\hline $\begin{array}{l}\text { Albumin, } \\
\text { mean (SD), } \\
\text { g/L }\end{array}$ & $42.15(7.08)$ & $41.13(5.04)$ & $39.32(4.00)$ & $37.76(7.74)$ & 0.121 & 0.016 \\
\hline $\begin{array}{l}\text { Bilirubin, } \\
\text { median } \\
(\mathrm{IQR}), \\
\mu \mathrm{mol} / \mathrm{L}\end{array}$ & $\begin{array}{l}11.50 \\
(9.70-15.78)\end{array}$ & $\begin{array}{l}12.90(10.25- \\
19.83)\end{array}$ & $\begin{array}{l}14.85(12.13- \\
22.45)\end{array}$ & $\begin{array}{l}15.30(11.60- \\
23.18)\end{array}$ & 0.123 & 0.034 \\
\hline $\begin{array}{l}\text { ALT, } \\
\text { median } \\
(\mathrm{IQR}), \mathrm{U} / \mathrm{L}\end{array}$ & $\begin{array}{l}18.50(11.25- \\
26.50)\end{array}$ & $\begin{array}{l}18.00(12.00- \\
22.75)\end{array}$ & $\begin{array}{l}12.50(11.00- \\
18.25)\end{array}$ & $\begin{array}{l}14.50(10.25- \\
24.00)\end{array}$ & 0.358 & 0.583 \\
\hline $\begin{array}{l}\text { AST, } \\
\text { median } \\
\text { (IQR), U/L } \\
\text { Kidney } \\
\text { function }\end{array}$ & $\begin{array}{l}22.00(18.00- \\
25.00)\end{array}$ & $\begin{array}{l}23.00(16.00- \\
32.00)\end{array}$ & $\begin{array}{l}21.00(17.00- \\
28.50)\end{array}$ & $\begin{array}{l}22.00(17.25- \\
28.25)\end{array}$ & 0.958 & 0.168 \\
\hline $\begin{array}{l}\text { Scr, median } \\
\text { (IQR), } \\
\text { mg/dl }\end{array}$ & $\begin{array}{l}0.67 \\
(0.55-0.86)\end{array}$ & $\begin{array}{l}0.80 \\
(0.68-0.92)\end{array}$ & $\begin{array}{l}0.80 \\
(0.65-1.01)\end{array}$ & $\begin{array}{l}0.78 \\
(0.62-1.05)\end{array}$ & 0.126 & 0.033 \\
\hline
\end{tabular}




\begin{tabular}{|c|c|c|c|c|c|c|}
\hline & $\begin{array}{l}\text { Group A } \\
(\mathrm{n}=20)\end{array}$ & $\begin{array}{l}\text { Group B } \\
(\mathrm{n}=20)\end{array}$ & $\begin{array}{l}\text { Group C } \\
(\mathrm{n}=20)\end{array}$ & $\begin{array}{l}\text { Group D } \\
(\mathrm{n}=20)\end{array}$ & $P$ value & $P$ trend \\
\hline $\begin{array}{l}\text { BUN, } \\
\text { median } \\
(\mathrm{IQR}) \\
\mathrm{mg} / \mathrm{dl}\end{array}$ & $\begin{array}{l}14.57(11.90- \\
16.95)\end{array}$ & $\begin{array}{l}15.55(12.74- \\
19.54)\end{array}$ & $\begin{array}{l}13.98(10.99- \\
17.44)\end{array}$ & $\begin{array}{l}15.69(11.25- \\
20.02)\end{array}$ & 0.746 & 0.761 \\
\hline $\begin{array}{l}\text { GFR, mean } \\
(\mathrm{SD}), \\
\mathrm{mL} / \mathrm{min} / 1.73 \mathrm{~m}^{2}\end{array}$ & $\begin{array}{l}136.83 \\
(29.07)\end{array}$ & $\begin{array}{l}115.41 \\
(26.87)\end{array}$ & $\begin{array}{l}107.24 \\
(28.67)\end{array}$ & $\begin{array}{l}111.98 \\
(35.09)\end{array}$ & 0.014 & 0.008 \\
\hline $\begin{array}{l}\mathrm{EF} \text {, mean } \\
(\mathrm{SD}) \%\end{array}$ & $63.65(2.37)$ & $62.35(1.90)$ & $64.05(3.32)$ & $63.10(3.16)$ & 0.239 & 0.986 \\
\hline
\end{tabular}

Table 1. Baseline characteristics of patients in different age groups.

BMI, body mass index; ALT, alanine aminotransferase; AST, aspartate aminotransferase; Scr, serum creatinine, BUN, blood urea nitrogen; GFR, glomerular filtration rate; EF, ejection fraction.

$P$ value in one-way analysis of variance, Kruskal-Wallis test, or $\chi^{2}$ test; $P$ trend in linear regression or Mantel-Haenszel $\chi^{2}$ test.

Table 2. Comparison of anesthetic effects in different age groups.

\begin{tabular}{lllllll}
\hline & $\begin{array}{l}\text { Group A } \\
(\mathrm{n}=20)\end{array}$ & $\begin{array}{l}\text { Group B } \\
(\mathrm{n}=20)\end{array}$ & $\begin{array}{l}\text { Group C } \\
(\mathrm{n}=20)\end{array}$ & $\begin{array}{l}\text { Group D } \\
(\mathrm{n}=20)\end{array}$ & $P$ value & $P$ trend \\
\hline $\begin{array}{l}\text { Propofol } \\
\text { requirement, }\end{array}$ & $1.35(0.20)$ & $1.15(0.20)^{*}$ & $1.01(0.21)^{+}$ & $\begin{array}{l}0.83(0.17) \\
++\S-\end{array}$ & $<\mathbf{0 . 0 0 1}$ & $<\mathbf{0 . 0 0 1}$ \\
$\begin{array}{l}\text { mean (SD), } \\
\mathrm{mg} / \mathrm{kg}\end{array}$ & & & & & \\
$\begin{array}{l}\mathrm{T} \text { reflection } \\
\text { disappear, } \\
\text { mean (SD), } \mathrm{s}\end{array}$ & $262.50(36.11)$ & $\begin{array}{l}230.00(42.43) \\
*\end{array}$ & $\begin{array}{l}200.50(43.34) \\
+\end{array}$ & $\begin{array}{l}165.00(32.36) \\
++\S-\end{array}$ & $<\mathbf{0 . 0 0 1}$ & $<\mathbf{0 . 0 0 1}$ \\
\hline
\end{tabular}

* Significant difference between Group A and B, + Significant difference between Group A and C, ++ Significant difference between Group A and D, § Significant difference between Group B and D, — Significant difference between Group C and D.

\begin{tabular}{|c|c|c|c|}
\hline & Propofol requirement, $\mathrm{mg} / \mathrm{kg}$ & Propofol requirement, $\mathrm{mg} / \mathrm{kg}$ & Propofol requirement, $\mathrm{mg} / \mathrm{kg}$ \\
\hline & $\mathrm{r}$ & $95 \% \mathrm{CI}$ & $P$ value \\
\hline Age, yr & -0.689 & $-0.789^{\sim}-0.553$ & $<0.001$ \\
\hline BMI, $\mathrm{kg} / \mathrm{m}^{2}$ & -0.214 & $-0.414 \sim 0.006$ & 0.057 \\
\hline Albumin, g/L & 0.312 & $0.099 \sim 0.497$ & 0.005 \\
\hline Ln (Bilirubin), $\mu \mathrm{mol} / \mathrm{L}$ & -0.169 & $-0.375 \sim 0.052$ & 0.133 \\
\hline $\mathrm{Ln}(\mathrm{ALT}), \mathrm{U} / \mathrm{L}$ & 0.018 & $-0.203 \sim 0.237$ & 0.875 \\
\hline $\mathrm{Ln}(\mathrm{AST}), \mathrm{U} / \mathrm{L}$ & -0.145 & $-0.353 \sim 0.078$ & 0.201 \\
\hline $\mathrm{Ln}(\mathrm{Scr}), \mathrm{mg} / \mathrm{dl}$ & -0.173 & $-0.379^{\sim} 0.048$ & 0.124 \\
\hline Ln (BUN), mg/dl & 0.037 & $-0.184 \sim 0.255$ & 0.744 \\
\hline $\mathrm{GFR}, \mathrm{mL} / \mathrm{min} / 1.73 \mathrm{~m}^{2}$ & 0.286 & $0.070^{\sim} 0.476$ & 0.010 \\
\hline $\mathrm{EF}, \%$ & 0.095 & $-0.127^{\sim} 0.308$ & 0.401 \\
\hline
\end{tabular}


Table 3 . Correlation coefficients between age and propofol requirement.

BMI, body mass index; ALT, alanine aminotransferase; AST, aspartate aminotransferase; Scr, serum creatinine, BUN, blood urea nitrogen; GFR, glomerular filtration rate; EF, ejection fraction.

Table 4 . Effects of age on propofol requirement.

\begin{tabular}{lllllll}
\hline Age, yr & Model I & Model I & Model II & Model II & Model III & Model III \\
\hline & $\beta(95 \%$ CI $)$ & $P$ value & $\beta(95 \%$ CI $)$ & $P$ value & $\beta(95 \%$ CI $)$ & $P$ value \\
Per 1 SD & $-0.187\left(-0.232^{\sim}-0.143\right)$ & $<\mathbf{0 . 0 0 1}$ & $-0.185\left(-0.230^{\sim}-0.140\right)$ & $<\mathbf{0 . 0 0 1}$ & $-0.170\left(-0.224^{\sim}-0.117\right)$ & $<\mathbf{0 . 0 0 1}$ \\
Group A & $0.00[$ reference] & & $0.00[$ reference] & & $0.00[$ reference] & \\
Group B & $-0.201\left(-0.323^{\sim}-0.078\right)$ & $\mathbf{0 . 0 0 2}$ & $-0.185\left(-0.310^{\sim}-0.060\right)$ & $\mathbf{0 . 0 0 4}$ & $-0.158\left(-0.289^{\sim}-0.027\right)$ & $\mathbf{0 . 0 1 9}$ \\
Group C & $-0.344\left(-0.466^{\sim}-0.221\right)$ & $<\mathbf{0 . 0 0 1}$ & $-0.348\left(-0.470^{\sim}-0.226\right)$ & $<\mathbf{0 . 0 0 1}$ & $-0.321\left(-0.457^{\sim}-0.184\right)$ & $<\mathbf{0 . 0 0 1}$ \\
Group D & $-0.525\left(-0.648^{\sim}-0.403\right)$ & $<\mathbf{0 . 0 0 1}$ & $-0.512\left(-0.637^{\sim}-0.387\right)$ & $<\mathbf{0 . 0 0 1}$ & $-0.467\left(-0.608^{\sim}-0.327\right)$ & $<\mathbf{0 . 0 0 1}$ \\
Trend & $<\mathbf{0 . 0 0 1}$ & $<\mathbf{0 . 0 0 1}$ & $<\mathbf{0 . 0 0 1}$ & $<\mathbf{0 . 0 0 1}$ & $<\mathbf{0 . 0 0 1}$ & $<\mathbf{0 . 0 0 1}$ \\
\hline
\end{tabular}

Model I: unadjusted; Model II: adjusted for gender and BMI; Model III: further adjusted for albumin, bilirubin, ALT, AST, and GFR. One SD is equal to 12.

BMI, body mass index; ALT, alanine aminotransferase; AST, aspartate aminotransferase; GFR, glomerular filtration rate.

\section{Figure legends}

Figure 1 . Adjusted dose-response relationship between propofol requirement and age. Adjusted for gender, BMI, albumin, bilirubin, ALT, AST, and GFR. BMI, body mass index; ALT, alanine aminotransferase; AST, aspartate aminotransferase; GFR, glomerular filtration rate.

Figure 2 . The changes of hemodynamic parameters at 5 different time points during the induction of anesthesia. (A ) Mean arterial pressure (MAP). (B ) Heart rate (HR). The percent changes of MAP and HR relative to the baseline between the 4 groups were compared. All the values are presented as mean. T0, before propofol administration; T1, LOC; T2, 3 minutes after the administration of fentanyl and cisatracurium; T3, 1 minute after intubation; T4, 5 minutes after intubation; T5, 10 minutes after intubation. 


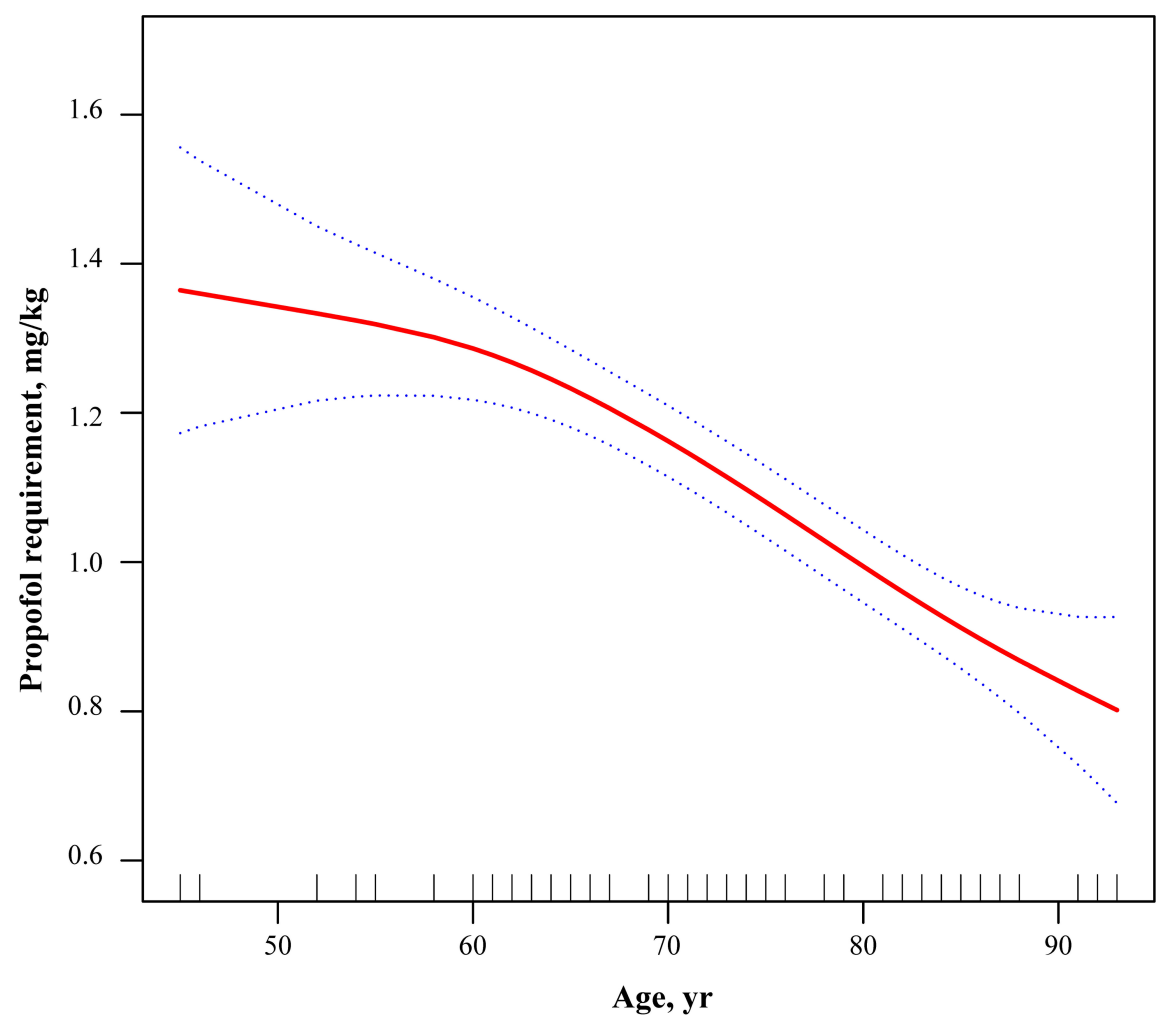



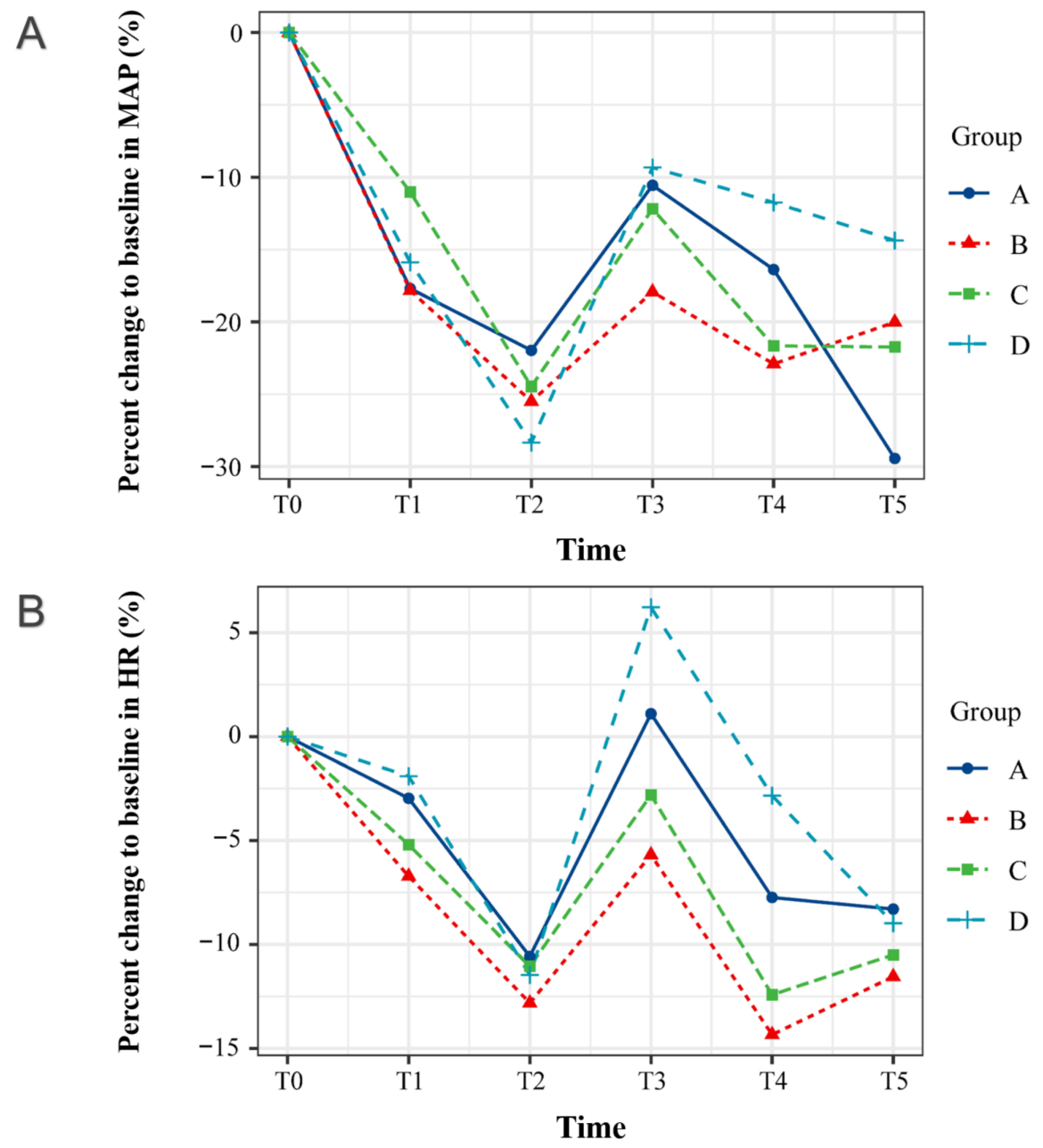